\title{
USP22-dependent HSP9OAB1 expression promotes resistance to HSP90 inhibition in mammary and colorectal cancer
}

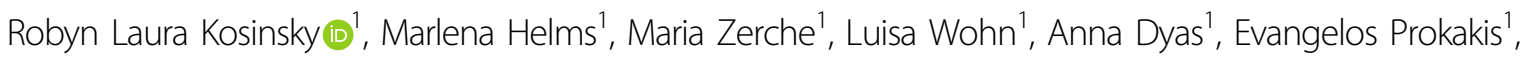 \\ Zahra Basir Kazerouni ${ }^{1}$, Upasana Bedi', Florian Wegwitz ${ }^{1}$ and Steven A. Johnsen $\mathbb{D}^{2}$
}

\begin{abstract}
As a member of the 11-gene "death-from-cancer" gene expression signature, overexpression of the Ubiquitin-Specific Protease 22 (USP22) was associated with poor prognosis in various human malignancies. To investigate the function of USP22 in cancer development and progression, we sought to detect common USP22-dependent molecular mechanisms in human colorectal and breast cancer cell lines. We performed mRNA-seq to compare gene expression profiles of various colorectal (SW837, SW480, HCT116) and mammary (HCC1954 and MCF10A) cell lines upon siRNAmediated knockdown of USP22. Intriguingly, while USP22 depletion had highly heterogeneous effects across the cell lines, all cell lines displayed a common reduction in the expression of Heat Shock Protein 90 Alpha Family Class $B$ Member 1 (HSP9OAB1). The downregulation of HSP90AB1 was confirmed at the protein level in these cell lines as well as in colorectal and mammary tumors in mice with tissue-specific Usp22 deletions. Mechanistically, we detected a significant reduction of H3K9ac on the HSP9OAB1 gene in USP22-deficient cells. Interestingly, USP22-deficient cells displayed a high dependence on HSP9OAB1 expression and diminishing HSP90 activity further using the HSP90 inhibitor Ganetespib resulted in increased therapeutic vulnerability in both colorectal and breast cancer cells in vitro. Accordingly, subcutaneously transplanted CRC cells deficient in USP22 expression displayed increased sensitivity towards Ganetespib treatment in vivo. Together, we discovered that HSP9OAB1 is USP22-dependent and that cooperative targeting of USP22 and HSP90 may provide an effective approach to the treatment of colorectal and breast cancer.
\end{abstract}

\section{Introduction}

Despite improved diagnostic and therapeutic approaches, malignant diseases remain a major health burden worldwide. In fact, studies indicate that cancer has replaced cardiovascular disorders as the leading cause of death in several countries ${ }^{1}$. This emphasizes the need to improve the current understanding of the development,

\footnotetext{
Correspondence: Robyn Laura Kosinsky (robyn-laura.kosinsky@zentr.unigoettingen.de) or Steven A. Johnsen (johnsen.steven@mayo.edu) 'Department of General, Visceral and Pediatric Surgery, University Medical Center Göttingen, Justus-von-Liebig-Weg 11, 37077 Göttingen, Germany ${ }^{2}$ Gene Regulatory Mechanisms and Molecular Epigenetics Lab, Division of Gastroenterology and Hepatology, Mayo Clinic, 200 First St SW, Rochester, MN, USA

Edited by D. Guardavaccaro
}

progression and recurrence of malignant diseases. Interestingly, the overexpression of a specific group of genes identified as the so-called 11-gene "death-from-cancer" expression signature was associated with particularly poor patient survival, distant metastasis and high recurrence rates $^{2,3}$. The Ubiquitin-Specific Protease 22 (USP22) is a member of this signature and has been described in numerous studies to be overexpressed in various human malignancies ${ }^{4}$. These studies were mainly based on immunohistochemical analyses or evaluations of mRNA. USP22, together with Ataxin 7 (ATXN7), Ataxin 7-Like 3 (ATXN7L3) and Enhancer of Yellow 2 Homolog (ENY2), forms the deubiquitinating module (DUBm) of the SAGA (Spt-Ada-Gcn5 Acetyltransferase) complex. In addition to

\section{(c) The Author(s) 2019}

(c) Open Access This article is licensed under a Creative Commons Attribution 4.0 International License, which permits use, sharing, adaptation, distribution and reproduction in any medium or format, as long as you give appropriate credit to the original author(s) and the source, provide a link to the Creative Commons license, and indicate if changes were made. The images or other third party material in this article are included in the article's Creative Commons license, unless indicated otherwise in a credit line to the material. If material is not included in the article's Creative Commons license and your intended use is not permitted by statutory regulation or exceeds the permitted use, you will need to obtain permission directly from the copyright holder. To view a copy of this license, visit http://creativecommons.org/licenses/by/4.0/. 
the DUBm, the SAGA transcriptional cofactor complex contains the histone acetyltransferase (HAT) General Control Nonderepressible 5 (GCN5), which promotes transcription via acetylation of lysine 9 of histone 3 $(\mathrm{H} 3 \mathrm{~K} 9 \mathrm{ac})^{5}$. Interestingly, in contrast to the prevailing view that USP22 is a universal oncogene, our recent results demonstrated a context-dependent tumor suppressor function of USP22 in colorectal cancer whereby loss of USP22 expression resulted in decreased SAGA-mediated H3K9ac on the PRKAA2 gene (which encodes the AMPactivated protein kinase-2) and a concomitant downregulation of its expression, thereby leading to activation of the mTOR signaling pathway ${ }^{6}$. Importantly, while loss of USP22 expression resulted in increased tumor growth and aggressiveness, activation of the mTOR pathway resulted in a synthetic vulnerability of USP22-deficient colorectal cancer cells to mTOR inhibitor treatment.

Mechanistically, USP22 was reported to deubiquitinate the core histone $\mathrm{H} 2 \mathrm{~B}$ at lysine $120^{7}$. The loss of this monoubiquitination (H2Bub1) has been associated with advanced tumor grade and poor patient survival in colorectal $(\mathrm{CRC})^{8}$ and breast cancer ${ }^{9}$ and, therefore, H2Bub1 has been considered as a tumor-suppressive epigenetic mark. Apart from its function in deubiquitinating H2B, USP22 was also reported to deubiquitinate and thereby stabilize several key oncogenic proteins including $\mathrm{MYC}^{7}$ and Sirtuin $1(\operatorname{SIRT} 1)^{10}$. Based on its function in deubiquitinating $\mathrm{H} 2 \mathrm{~B}$ and oncogenic proteins, increased USP22 levels were reported to accelerate colorectal ${ }^{11-14}$ and breast cancer development and progression ${ }^{15,16}$. Thus, USP22 has been proposed as an attractive therapeutic target in malignant diseases and, indeed, there is ongoing research to generate and optimize USP22 inhibitors ${ }^{4}$, although caution must be used given our findings of the context-dependent function of USP22 in cancer.

In this study, we aimed to investigate the function of USP22 in colorectal and breast cancer and to detect common USP22-dependent molecular mechanisms which may be exploited for cancer treatment. For this purpose, we performed next-generation sequencing in several human cell lines and employed genetic tumor mouse models with intestine- and mammary-specific deletions of Usp22. Finally, after identifying HSP90AB1 as a novel USP22-dependent target gene, we evaluated the therapeutic targetability of USP22-deficient tumor cells in vitro and in vivo.

\section{Materials and methods}

\section{Cell culture and siRNA-mediated knockdowns}

Human cell lines were grown in their respective growth media supplemented with $10 \%$ fetal bovine serum, 100 units $/ \mathrm{ml}$ penicillin and $100 \mu \mathrm{g} / \mathrm{ml}$ streptomycin at $37^{\circ} \mathrm{C}$ and 5\% $\mathrm{CO}_{2}$ (SW837: DMEM/F-12, SW480: RPMI Glutamax, HCT116: McCoy's, HCC1954: RPMI Glutamax,
MCF10A: DMEM/F-12 supplemented with 5\% horse serum, $\quad 0.5 \mu \mathrm{g} / \mathrm{ml}$ hydrocortisone, $10 \mu \mathrm{g} / \mathrm{ml}$ Insulin, $20 \mathrm{ng} / \mathrm{ml}$ human epithelial growth factor, $0.1 \mu \mathrm{g} / \mathrm{ml}$ cholera toxin). siRNA (GE Dharmacon siGENOME; Table S1) transfections using a non-targeting control (NT5) or targeting USP22 or GCN5 were performed using Lipofectamine $^{\circledast}$ RNAiMAX (Invitrogen) according to the manufacturer's instructions. To test the effect of HSP90 inhibition cells were treated with the indicated concentrations of Ganetespib (Selleckchem) $24 \mathrm{~h}$ after siRNA-mediated knockdown for an additional $48 \mathrm{~h}$. As a negative control, DMSO was added to the cells.

\section{CRISPR/Cas9-mediated deletion of USP22}

The HCT116 colorectal cancer cell lines harboring a permanent deletion of the USP22 gene were described previously $^{6}$. Briefly, two single guide RNAs (sgRNAs) targeting USP22 (sgRNA1: 5'-CACCGGTGTTTGGC AGCTCATGCCC-3', sgRNA2: 5'-CACCGTTAGAGAG ACCTGGCGGTGG-3') were cloned into the pSpCas9 (BB)-2A-GFP (PX458, Addgene) vector containing Cas9 and GFP sequences. Single highly fluorescent cells were sorted into 96-well plates using fluorescence activated cell sorting (FACS) and single cell clones were expanded and the loss of USP22 was confirmed by western blot and qRT-PCR. To avoid potential off-target effects, two HCT116 USP22 $2^{-/-}$single cell clones were characterized and one non-targeted $U S P 22^{+/+}$single cell clone as well as the HCT116 parental cell line have been used as controls. Unless indicated otherwise, results obtained from one representative clone are shown.

\section{Proliferation assays}

To determine cell proliferation, 2000 HCT116 and 3000 HCC1954 cells were seeded onto 96-well assay plates (Corning Life Sciences) and confluence was measured daily using a Celigo ${ }^{\circledast}$ S Adherent Cell Cytometer (Nexcelom Bioscience LLC). The effect of Ganetespib treatment on cell confluence was tested by seeding 20,000 HCT116 and 30,000 HCC1954 cells per well of a 24-well plate and staining cells using $1 \%(\mathrm{w} / \mathrm{v})$ crystal violet in $20 \%$ ethanol after $48 \mathrm{~h}$ of HSP90i treatment.

\section{Western blotting and qRT-PCR}

Protein isolation, western blot analysis, RNA extraction, reverse transcription, and qRT-PCR were performed as previously described ${ }^{17}$. The primary antibodies for western blot and primers for qRT-PCR are listed in Tables S2 and $\mathrm{S} 3$, respectively.

\section{Chromatin immunoprecipitation}

Chromatin immunoprecipitation (ChIP) was performed to assess H3K9ac occupancy on the HSP9OAB1 gene. HCT116 USP $22^{+/+}$and USP22 $2^{-/-}$cells were fixed in $1 \%$ 
formaldehyde in PBS for $20 \mathrm{~min}$ and quenched with $125 \mathrm{mM}$ glycine for $5 \mathrm{~min}$. After washing cells twice with ice-cold PBS, they were scraped and washed with Nelson Buffer (150 mM NaCl, $20 \mathrm{mM}$ EDTA (pH 8.0), $50 \mathrm{mM}$ Tris/HCl (pH 7.5), 0.5\% (v/v) NP-40, 1\% (v/v) Triton-X$100,20 \mathrm{mM} \mathrm{NaF}$ and protease inhibitors). The pellets were resuspended in Lysis Buffer (20 mM EDTA, $150 \mathrm{mM}$ $\mathrm{NaCl}, 1 \%(\mathrm{v} / \mathrm{v}) \mathrm{NP}-40,0.1 \%(\mathrm{w} / \mathrm{v}) \mathrm{SDS}, 0.5 \%(\mathrm{w} / \mathrm{v})$ sodium deoxycholate, $20 \mathrm{mM} \mathrm{NaF}, 50 \mathrm{mM}$ Tris/ $\mathrm{HCl}(\mathrm{pH} 8.0)$ and protease inhibitors) and sonicated for 15 cycles $(30 \mathrm{~s} \mathrm{on} /$ off). After preclearing with 50\% Sepharose 4B (GE Healthcare) for $1 \mathrm{~h}$, samples were incubated with antibodies at $4{ }^{\circ} \mathrm{C}$ overnight (H3K9ac: C15410004, Diagenode; IgG: C15410206, Diagenode). Sepharose beads with Protein A (GE Healthcare) were added and incubated at $4{ }^{\circ} \mathrm{C}$ for $2 \mathrm{~h}$. Samples were washed twice with Wash Buffer (20 mM EDTA, $500 \mathrm{mM} \mathrm{LiCl,} \mathrm{1 \%} \mathrm{(v/v)} \mathrm{NP-40,} 20 \mathrm{mM}$ $\mathrm{NaF}, 1 \%(\mathrm{w} / \mathrm{v})$ sodium deoxycholate, $100 \mathrm{mM}$ Tris/HCl (pH 8.5)) and twice with TE buffer. Next, immune complexes were incubated with RNAse A (Qiagen) in $10 \mathrm{mM}$ Tris/ $\mathrm{HCl}(\mathrm{pH} 8.0)$ at $37^{\circ} \mathrm{C}$ for $30 \mathrm{~min}$ and de-crosslinked in $20 \mathrm{mM}$ EDTA, $2 \%(\mathrm{w} / \mathrm{v})$ SDS, $100 \mathrm{mM}$ Tris/HCl (pH 8) and $20 \mu \mathrm{g}$ Proteinase $\mathrm{K}$ at $65{ }^{\circ} \mathrm{C}$ overnight. DNA was extracted with Roti $^{\circledast}$ phenol/chloroform/isoamylalcohol (Roth) and precipitated with ethanol. Input samples were used as controls and primer sequences for subsequent qRT-PCR are listed in Table S4.

\section{Library preparation and next-generation sequencing}

To evaluate transcriptome-wide effects of USP22 loss, mRNA-seq and subsequent analysis was performed. SW837, SW480, HCT116, MCF10A and HCC1954 cells were transfected with control or USP22 siRNAs $(n=3-4$ per condition). RNA integrity was verified using agarose gel electrophoresis and libraries were prepared using TruSeq RNA Library Preparation Kit v2 (Illumina ${ }^{\circledR}$ ) or NEXTflex Rapid Directional RNA-Seq Kit (Biooscientific). Sequencing was performed at the Transcriptome and Genome Analysis Laboratory (TAL, Göttingen) using HiSeq 2000 or 4000 (Illumina $^{\circledR}$ ). All mRNA-seq data have been deposited at ArrayExpress (http://www.ebi.ac.uk/ arrayexpress, accession numbers: E-MTAB-7393 (HCT116) $)^{6}$ E-MTAB-8214 (SW480), E-MTAB-8215 (SW837), E-MTAB-8247 (MCF10A), E-MTAB-8256 (HCC1954)). Data analysis and Gene Set Enrichment Analysis $\left(\mathrm{GSEA} ;{ }^{18}\right.$ ), were performed as previously described $^{17,19}$.

\section{Generation of mice and genotyping}

All animal work was performed according to institutional regulations for care and use of laboratory animals and approved by the Lower Saxony State Office for Consumer Protection and Food Safety (LAVES; registration numbers: G15/2039, G15/1754 and G11/540).
$U_{s p} 22^{\text {flox }}$ mice were generated as described previously ${ }^{6}$ by removing lac $Z$ and neomycin resistance loci from the Usp $22^{\text {tmla(KOMP)Wtsi }}$ C57BL6 mouse line generated from embryonic stem cells obtained from the University of California-Davis Knockout Mouse Project Repository ${ }^{20}$ by FLP-mediated excision ${ }^{21}$. Usp $22^{\text {flox }}$ mice were crossed with MMTV-Cre (a kind gift from L. Henninghausen, National Institutes of Health, USA) and Tg(MMTVErbB2)NK1Mul/J (The Jackson Laboratory) animals (FVB/N background) to achieve a mammary-specific Usp 22 knockout and to promote tumorigenesis, respectively. Moreover, Usp $22^{\text {flox }}$ mice were crossed with VillinCre ${ }^{\mathrm{ERT} 2}$ and $A p c^{1638 \mathrm{~N}}$ mouse lines (C57BL/6N background) to achieve an intestinal knockout and to investigate its role in tumorigenesis ${ }^{6}$. The $\mathrm{Apc}^{1638 \mathrm{~N} /+}$ mouse line was a kind gift from F. Bosman (Erasmus University Medical Center Rotterdam, The Netherlands). Multiple replicates $(n=3-6)$ were utilized in order to ensure reproducibility of findings.

\section{Tamoxifen treatment}

4-week-old Villin-Cre ${ }^{\text {ERT2 }}, \quad$ Usp22 $2^{\text {flox }}$, and Villin$C r e^{\mathrm{ERT} 2}, A p c^{1638 \mathrm{~N} /+}, U_{s p} 22^{\text {flox }}$ mice were injected with a total dose of $1 \mathrm{mg}$ Tamoxifen (Sigma-Aldrich) per day for five consecutive days to induce an intestinal knockout of Usp 22. Tamoxifen (5\% w/v) was dissolved in $100 \%$ ethanol and mixed 1:10 with autoclaved sunflower oil right before intraperitoneal injection.

\section{Xenograft experiments}

Immunodeficient SCID mice were housed in a controlled, germ-free environment. Animals were randomized into treatment groups and anesthetized by isoflurane inhalation (2-3\%, Forene) and HCT116 USP22 ${ }^{+/+}$and

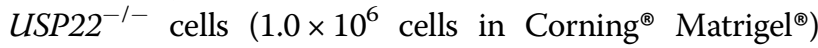
were injected subcutaneously into the left and right flank, respectively, using a $0.3 \mathrm{ml}$ Micro-Fine syringe (BD Bioscience). The bodyweight, as well as the size of the growing tumors, were monitored daily. After the tumors reached a volume of approximately $100 \mathrm{~mm}^{3}, 25 \mathrm{mg} / \mathrm{kg}$ Ganetespib (AdooQ) or vehicle solution (10\% DMSO (v/v), $20 \%$ (v/v) pre-warmed Cremophor RH40 $\left(55^{\circ} \mathrm{C}\right.$; SigmaAldrich), 5\% (w/v) dextrose) was injected i.v. for three consecutive days and after 4 days break, for 3 further days. Experiments were performed with six replicates per group in order to assure reproducibility. Experiments were performed blinded during tumor size measurement and subsequent immunohistochemical staining.

\section{H\&E staining and immunohistochemistry (IHC)}

Histological staining was performed as described previously ${ }^{20}$. Briefly, $5 \mu \mathrm{m}$ tissue sections were deparaffinized in xylol for $20 \mathrm{~min}$, rehydrated using decreasing ethanol concentrations $(100 \%, 90 \%, 70 \%)$ for 
5 min each and washed with water. For H\&E staining, slides were stained in Mayer's hematoxylin solution (Roth) for $5 \mathrm{~min}$ and counterstained with eosin for 5-10 min. For IHC, sections were boiled in $10 \mathrm{mM}$ citric acid buffer for $15 \mathrm{~min}$ and incubated with $5 \% \mathrm{H}_{2} \mathrm{O}_{2}$ in PBS. Upon blocking with $10 \%$ fetal bovine serum (FBS) in PBS, sections were incubated with primary antibodies (Table S2) diluted in 5\% FBS in PBS in a humid chamber at $4{ }^{\circ} \mathrm{C}$ overnight. Biotinylated secondary antibodies (1:200; GE Healthcare) and ExtrAvidin-Peroxidase (1:1,000; Sigma-Aldrich) diluted in PBS were added each for $1 \mathrm{~h}$. Staining was developed using 3,3'-diaminobenzidin-tetrahydrochloride (DAB; Roth) and counterstaining was performed using hematoxylin. Slides were dehydrated, incubated in xylol and mounted. To quantify HSP90AB1 IHC staining intensity we utilized the "color deconvolution" tool in FIJI to separate the DAB signal from the hematoxylin/background signals. The maximum intensity was divided by the minimum intensity in 4-6 images per staining per mouse.

\section{Statistical analyses}

All graphs have been designed and the area under the curve (AUC) has been calculated with GraphPad Prism version 5.04 (GraphPad Software, Inc.). Statistical analysis was performed using Student's $t$-test or one-way ANOVA and subsequent Tukey Post hoc test $(\alpha=0.05)$. Variation between different groups that were statistically compared was similar.

\section{Results}

\section{USP22-deficiency decreases mRNA levels of heat shock} factor HSP9OAB1

While our recent work suggested that USP22 can have context-dependent effects in cancer and have either tumor-promoting or suppressing functions ${ }^{6}$, previous studies have largely reported that overexpression of USP22 is associated with a more aggressive tumor phenotype. Notably, human tumor gene expression data generated by the TCGA Research Network (http:// cancergenome.nih.gov/ $;^{22}$ ) implied that a significant proportion of colorectal cancer (22\%) and breast cancer patients (26\%) display low USP22 expression (Fig. S1A). Thus, we sought to obtain a broad overview of the transcriptome-wide consequences of USP22 depletion in various colorectal and mammary cancer cell lines in vitro. Therefore, we performed siRNA-mediated knockdowns of USP22 (siUSP22) and compared these to control knockdowns (siControl) via mRNA-seq analysis in five different human cell lines. These cells originate from rectum adenocarcinoma (SW837), colorectal adenocarcinoma (SW480), colorectal carcinoma (HCT116), nontransformed mammary epithelium (MCF10A) and HER2-positive mammary ductal carcinoma (HCC1954).
As expected based on our previous findings, a high degree of heterogeneity was observed in the effects elicited by USP22 depletion in the various cell lines. While no mutually upregulated genes were detected (Fig. 1a), we identified eight mutually downregulated genes including the Heat Shock Protein 90 encoding gene HSP90AB1 as well as two HSP90 pseudogenes HSP90AB2P and $H S P 90 A B 3 P$ (Fig. 1b). Indeed, the reduction of HSP9OAB1 mRNA levels was confirmed using qRT-PCR in all five cell lines, yet not statistically significant in MCF10A cells (Fig. 1c). Together, upon the transient depletion of USP22 we detected a downregulation of $H S P 90 A B 1$ by up to $60 \%$ in five human cell lines of various origins.

\section{USP22-deficiency influences HSP90-dependent signaling}

HSP90AB1 functions as a constitutively active molecular chaperone and was found to be overexpressed in various malignant diseases ${ }^{23}$. Therefore, over the last decades, numerous HSP90 inhibitors (HSP90i) have been developed and tested in clinical trials ${ }^{24}$. Interestingly, when elucidating gene expression signatures enriched following USP22 depletion by performing Gene Set Enrichment Analysis (GSEA), while the individual gene expression changes between cell lines were highly variable, we were able to detect an enrichment of genes downregulated upon the treatment with the HSP90i 17AAG in HCT116 (Fig. 2a) and HCC1954 cells (Fig. 2b). Among these genes were 24 which were mutually regulated in both cell lines. These genes included ATP-Binding Cassette sub-family E member 1 (ABCE1), Cytochrome C Somatic (CYCS) and Eukaryotic Translation Initiation Factor $4 A 1$ (EIF4A1) (Fig. 2c). To obtain further mechanistic insights, we utilized HCT116 cells with a permanent CRISPR/Cas9-mediated deletion of the USP22 gene $^{6}$. Consistent with siRNA-mediated knockdown, these cells displayed a reduction of $H S P 90 A B 1$ at the mRNA level (Supplementary Fig. S1B). Next, to confirm the dependency of $A B C E 1, C Y C S$ and EIF4A1 expression on HSP90 as well as USP22, HCT116 wild type and USP22 knockout cells were treated with the HSP90i Ganetespib. Indeed, Ganetespib treatment or USP22 deletion resulted in reduced mRNA expression of these genes (Fig. 2d). Since USP22 functions to regulate transcription as a member of the SAGA complex, we next performed ChIP for H3K9ac, a histone modification mediated by the SAGA acetyltransferase GCN5, on the HSP90AB1 gene in HCT116 cells. Notably, consistent with a direct role of USP22 and the SAGA complex, H3K9ac occupancy on the HSP90AB1 gene was significantly decreased in USP22 knockout cells compared to wild type cells (Fig. 2e). As a control, a H3K9ac-negative site on the same gene was examined. Consistent with a general role of SAGA in the regulation of $H S P 90 A B 1$ mRNA expression, HSP90AB1 mRNA levels were also 
A siUSP22: Upregulated

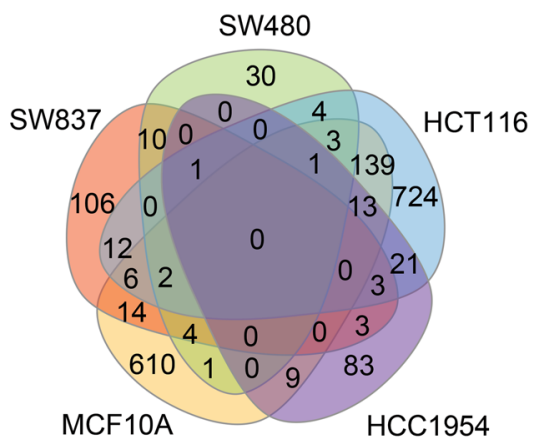

B

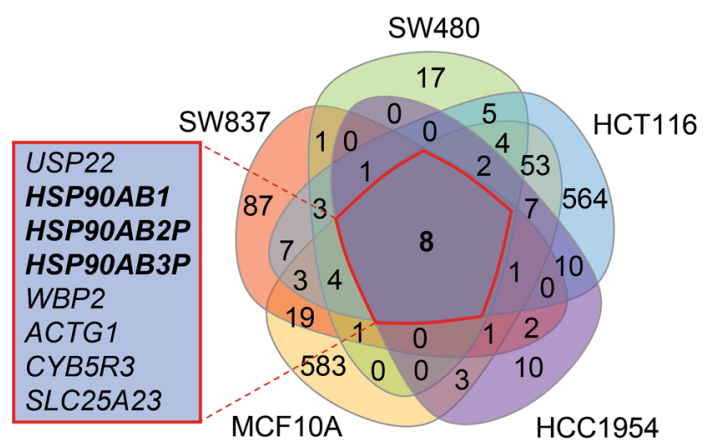

C

Relative USP22 mRNA expression
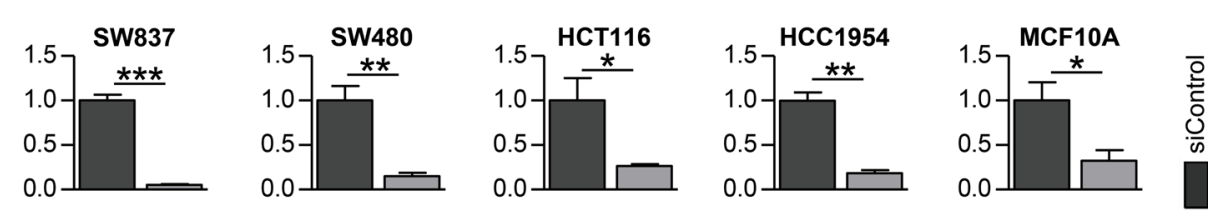

Relative HSP90AB1 mRNA expression
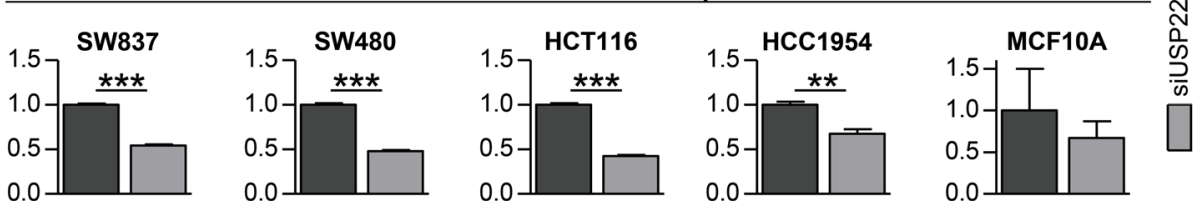

Fig. 1 USP22 loss reduces HSP90AB1 mRNA expression. a siRNA-mediated USP22 knockdowns were performed in SW837, SW480, HCT116, MCF10A, and HCC1954 cells $(n=3-4)$. While no overlap was detected in the upregulated genes, $\mathbf{b}$ all five human cell lines displayed reduced expression of the heat shock factors HSP90AB1, HSP90AB2P, and HSP90AB3P. c As verified using qRT-PCR in three independent experiments, the siRNAmediated depletion of USP22 resulted in reduced HSP9OAB1 mRNA levels. Mean \pm SEM, Student's $t$-test.

reduced following siRNA-mediated depletion of GCN5 (Fig. 2f, Supplementary Fig. S1C). In summary, in USP22deficient HCT116 cells, GCN5-mediated H3K9ac occupancy on $H S P 90 A B 1$ is reduced, thereby resulting in decreased HSP90AB1 mRNA expression and downregulation of HSP90-dependent genes.

\section{USP22 loss reduces HSP90AB1 protein levels in vitro and} in vivo

To verify that the downregulation of $H S P 90 A B 1$ mRNA expression results in reduced HSP90AB1 protein levels upon depletion of USP22, we performed western blot analysis in HCT116 and HCC1954 cells. Consistent with the effects observed on mRNA levels, we also observed a decrease in HSP90AB1 protein levels in USP22 knockdown cells (Fig. 3a, b). We next sought to substantiate these findings in vivo via immunohistochemistry for HSP90AB1 in tissue sections from Usp22-deficient murine models. For this purpose, we utilized our previously published Villin-Cre ${ }^{\mathrm{ERT} 2}$, Usp $22^{\text {flox }}$ mouse model containing an intestine-specific, conditional Usp22 knockout $^{6}$. Here, normal colorectal epithelium displayed reduced HSP90AB1 staining (Fig. 3c, d). Importantly, tumors derived from Villin-Cre ${ }^{\mathrm{ERT} 2}, U_{s p} 22^{\text {flox }}$ mice containing a mutation in the Adenomatous Polyposis Coli $(A P C)$ tumor suppressor gene $\left(\mathrm{APC}^{1638 \mathrm{~N} /+}\right.$ ) similarly displayed decreased HSP90AB1 levels compared to the Usp 22 wild type control tumors. Finally, this finding could be further confirmed in normal mammary epithelium and mammary tumors derived from mice containing a MMTV-Cre driven mammary-specific deletion ${ }^{25}$ of Usp 22 in a Her2-driven model of breast cancer. Together, these findings suggest that USP22 is required for high level expression of HSP90AB1 protein levels both in human cancer cell lines in vitro as well as in murine epithelium and tumors in vivo.

\section{USP22 deficiency sensitizes CRC and breast cancer cells towards HSP90i}

Based on the finding that USP22 loss reduces HSP90AB1 levels, we hypothesized that the residual HSP90 levels might be essential for the survival and stress-resistance of USP22-depleted cells. Therefore, we further hypothesized that USP22-deficient cells may 

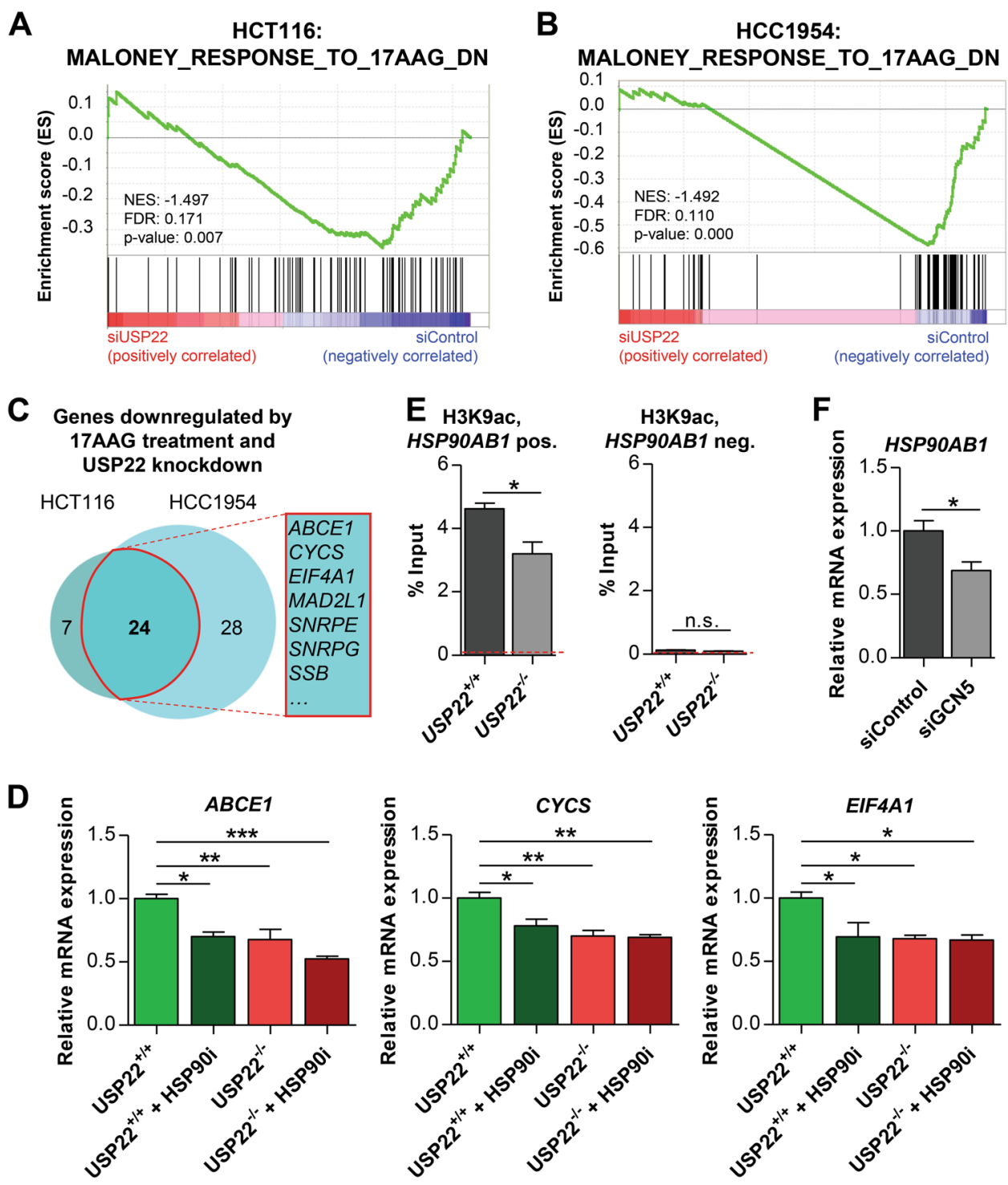

Fig. 2 HSP90-dependent signaling is affected by USP22. a GSEA indicated that genes, which are downregulated upon 17-AAG treatment are enriched in siUSP22 HCT116 and b HCC1954 cells. c Venn diagram displaying the overlap of HSP90i- and USP22 knockdown-responsive genes in HCT116 and HCC1954 cells. d The dependency of ABCE1, CYCS and EIF4A1 on HSP90 as well as USP22 was verified by treating HCT116 USP22 $2^{+/+}$and USP22 $2^{-\prime-}$ cells with the HSP90i Ganetespib or DMSO as a negative control. Mean \pm SEM, one-way ANOVA. e ChIP-qPCR revealed in two independent experiments that the H3K9ac occupancy on the HSP9OAB1 gene is significantly reduced upon the deletion of USP22 in HCT116 cells ( $n=4)$. An adjacent H3K9ac-negative site was analyzed as a control. The average signal for the negative control lgG is represented by dotted lines. Mean \pm SEM, $t$-test. f siRNA-mediated silencing of the acetyltransferase GCN5 reduced HSP90AB1 mRNA levels in HCT116 cells $(n=3)$. Mean \pm SEM, $t$-test.

therefore be particularly sensitive to the inhibition of the remaining HSP90 activity. Therefore, we treated control and USP22-depleted HCT116 and HCC1954 cells with increasing concentrations of Ganetespib for $48 \mathrm{~h}$ and visualized cell number via crystal violet staining. Indeed, both cell lines displayed increased sensitivity towards Ganetespib following USP22 knockdown (Fig. 4a). These results were further verified by measuring cellular confluence under the same experimental conditions using a Celigo $^{\circledR}$ S Adherent Cell Cytometer (Fig. 4b-e).
Moreover, this effect was not a non-specific effect due to siRNA transfection since the increased sensitivity toward Ganetespib could be confirmed in HCT116 cells containing a genetic deletion of the USP22 gene (Supplementary Fig. S1D). Moreover, protein lysates isolated from USP22 wild type and knockout cells were subjected to western blot analysis. In agreement with our hypothesis, Ganetespib-treated USP22 knockout cells displayed increased induction of the apoptosis marker cleaved PARP (Supplementary Fig. S1E). In summary, these 

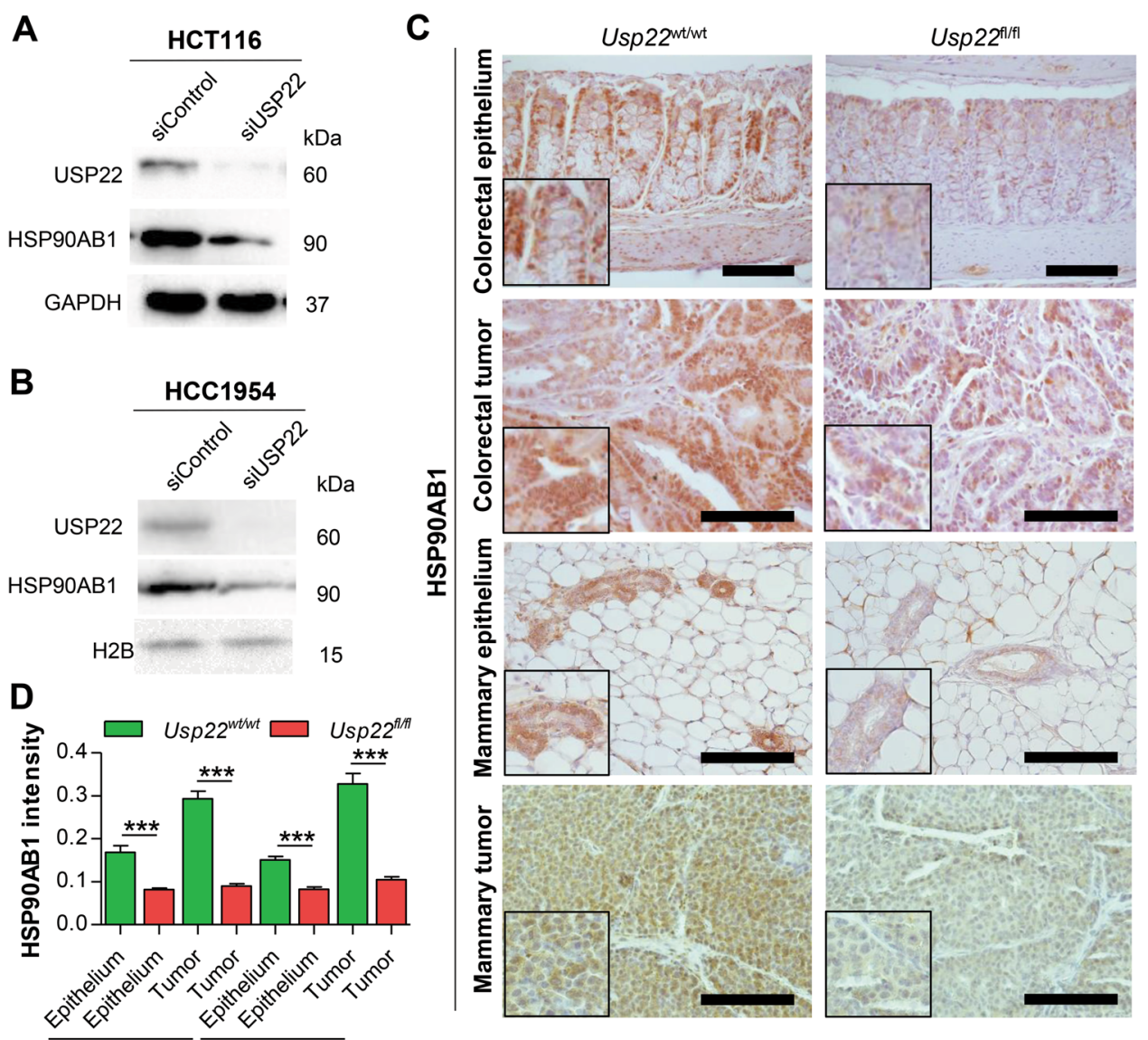

Fig. 3 USP22 loss results in reduced HSP90AB1 protein levels. a Western blot analysis was performed to demonstrate the reduction of HSP90AB1 protein levels after USP22 knockdown in HCT116 and b HCC1954 cells $(n=3)$. $\mathbf{c}$ Villin-Cre ${ }^{\text {ERT2 }}$, Usp22 $2^{\text {flox }}$ animals were generated to delete Usp22 specifically in the intestinal epithelium. In comparison, colorectal tumors from Villin-Cre ${ }^{\text {ERT2 }}, A p c^{1638 \mathrm{~N} /+}$, Usp22 flox mice and mammary tumors from MMTV-Her2, MMTV-Cre, Usp22 $2^{\text {flox }}$ animals were examined. As demonstrated using IHC, Usp22 deletion resulted in decreased HSP90AB1 staining in both intestinal $(n=6)$ and mammary epithelium $(n=3)$ as well as in colorectal $(n=6)$ and mammary tumors $(n=5)$. Scale bar: $100 \mu \mathrm{m}$. $\mathbf{d}$ HSP90AB1 IHC staining intensity was quantified using FIJ in 4-6 images per staining per mouse. Mean \pm SEM, $t$-test.

findings demonstrate that USP22-deficiency increases cellular sensitivity to HSP90i in vitro.

\section{USP22-deficient tumor cells display increased sensitivity to HSP90 inhibition}

Despite the fact that the majority of studies report oncogenic properties of USP22, we and others have discussed that USP22 might display tumor-suppressive functions in some biological contexts ${ }^{6,26}$. Thus, targeting USP22 could potentially have tumor-promoting effects. However, USP22-deficiency may introduce a tumorspecific therapeutic vulnerability that makes these tumors attractive targets for HSP90i treatment. To test this hypothesis in vivo, we utilized a xenograft-based approach to test the effect of Ganetespib treatment on USP22-deficient tumors in comparison to wildtype tumors. To this end, we injected USP22-deficient or wild type HCT116 cells subcutaneously into immunodeficient mice. Consistent with our previous results ${ }^{6}$, the loss of USP22 accelerated HCT116 tumor growth. Importantly, intravenous injection of Ganetespib had a dramatic and preferential effect on USP22 knockout cells where it had little effect on the growth of USP22-proficient tumors but significantly impaired the growth of USP22-deficient tumors more than $70 \%$ to a size $50 \%$ smaller than Ganetespib-treated wildtype tumors (Fig. 5a-c, Supplementary Fig. S1F). This finding was supported by immunohistochemical detection of the proliferation marker Ki67 (Fig. 5d, e). While the number of positively stained nuclei was increased after USP22 loss, Ganetespib treatment significantly reduced proliferation and elevated apoptosis (Supplementary Fig. S1G, H) preferentially in USP22-deficient tumors. Taken together, our results suggest that USP22 loss introduces a therapeutic vulnerability by sensitizing tumors towards HSP90 inhibition in vivo. 

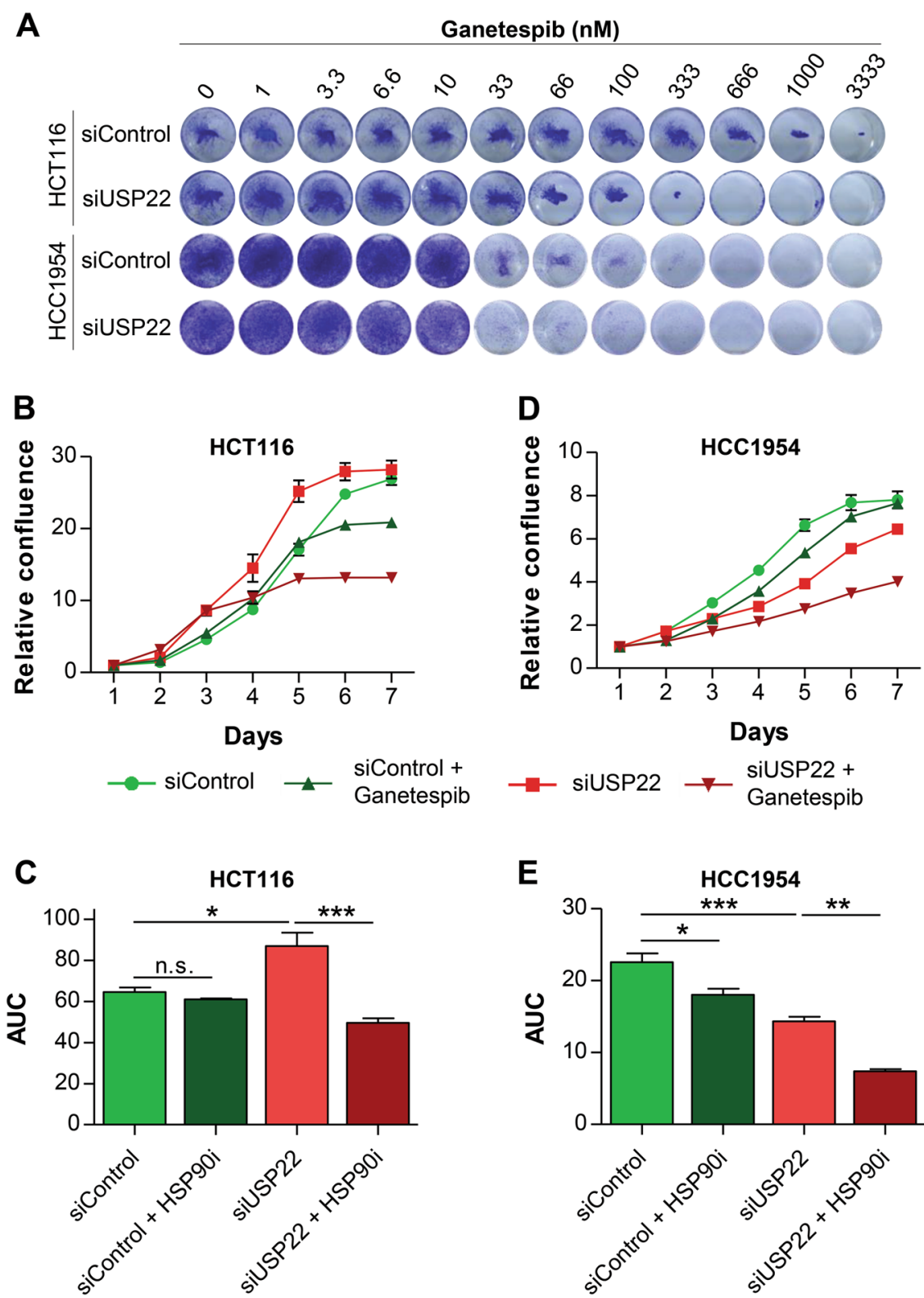

Fig. 4 USP22 deficiency sensitizes cancer cells towards HSP90 inhibition. a HCT116 and HCC1954 control and USP22-depleted cells were treated with increasing concentrations of the HSP90i Ganetespib for $48 \mathrm{~h}$ and visualized using crystal violet. USP22-depleted cells showed increased sensitivity towards Ganetespib. b The confluence of USP22 knockdown cells upon Ganetespib treatment was decreased in HCT116 cells as revealed by daily measurements of cell confluence $(n=6)$ as well as by c subsequent calculation of the area under the curve (AUC). $\mathbf{d}$, e This finding was confirmed in HCC1954 cells $(n=6)$. Mean \pm SEM, one-way ANOVA.

\section{Discussion}

In the literature, USP22 was described to exert protumorigenic functions in a wide variety of human malignancies and, therefore, USP22 would represent a promising therapeutic target. Thus, researchers are attempting to generate and optimize USP22 inhibitors ${ }^{4}$. In this study, we sought to build upon our previous results ${ }^{6}$ and identify common USP22-dependent molecular mechanisms in CRC and breast cancer. Intriguingly, we were able to demonstrate that HSP90AB1 levels are reduced upon USP22 loss in both colorectal and breast cancer cells in vitro. Interestingly, only the constitutively expressed HSP90 $\beta$ member HSP90AB1 was downregulated, while expression of the stress-inducible HSP90 $\alpha$ isoforms were not affected.

Our findings support a direct role for USP22 as an essential component of the SAGA complex in promoting the expression of $H S P 90 A B 1$ during cancer development 

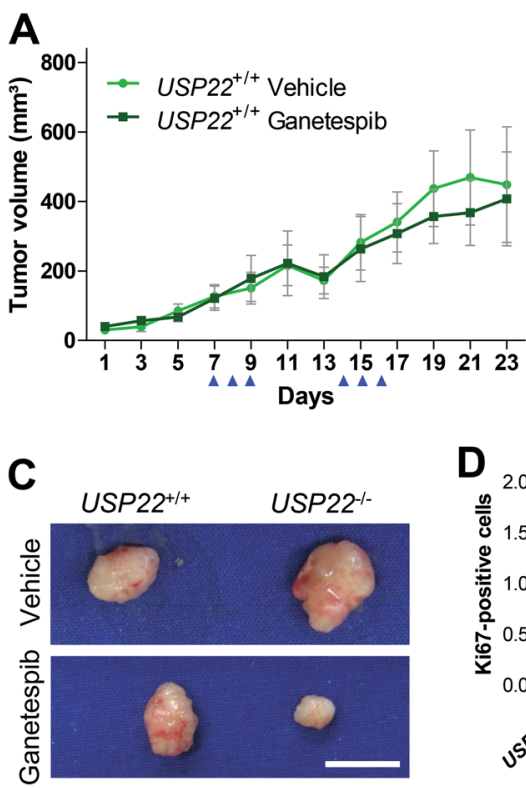

D

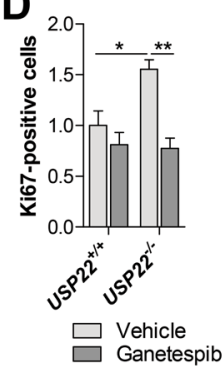

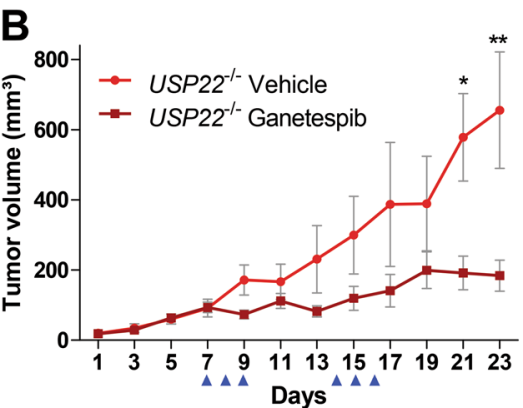

E

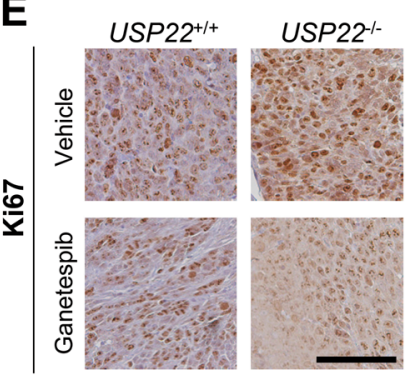

Fig. 5 USP22 deficiency conveys therapeutic vulnerability towards HSP90i in vivo. a In a xenograft approach, USP22 $2^{+/+}$and USP22 $2^{-/-}$HCT116 cells were injected subcutaneously into immunodeficient mice and treated with Ganetespib or vehicle ( $n=6$ per group). Results obtained from one representative wild type and USP22 knockout clone are shown. While Ganetespib treatment displayed minor effects on USP22 wild type tumors, $\mathbf{b}$ the accelerated growth of USP $22^{-1-}$ tumors was significantly impaired by HSP90 inhibition to levels below that of treated USP22 $2^{+/+}$tumors. Blue arrow heads indicate the days on which Ganetespib was administered. Mean \pm SEM, Student's t-test. c Representative images of USP22 $2^{+/+}$and USP22 $2^{-1-}$ tumors after the treatment with Ganetespib or vehicle. Scale bar: $1 \mathrm{~cm}$. d, e As detected by $I H C$, the number of proliferating Ki67-positive cells in USP22 $2^{-1-}$ tumors upon HSP90 inhibition was significantly decreased compared to tumors isolated from vehicle-treated mice. Mean \pm SEM, one-way ANOVA. Scale bar: $100 \mu \mathrm{m}$.

and progression. Increased expression of HSP90 family members has been associated with disease progression in the majority of human malignancies ${ }^{27}$ and HSP90AB1 has been described to play miscellaneous roles in human diseases due to the wide variety of client proteins ${ }^{23}$. Interestingly, while HSP90 is required for the stabilization of a number of oncogenes ${ }^{28}$, our findings suggest that the expression of its upstream regulator USP22 displays a dichotomous relationship with tumor progression where high USP22 expression correlates with unfavorable prognosis in breast cancer but improved survival in rectal cancer patients. Indeed, this was reflected in our proliferation assays in which the siRNA-mediated knockdown reduced the confluence of HCC1954 cells and promoted growth of HCT116 cells. Surprisingly, but consistent with these findings, analysis of publicly available TCGA data published on the Human Protein Atlas website $^{22}$ demonstrate that high HSP90AB1 expression is also associated with poor prognosis in mammary carcinoma but a more favorable outcome in rectal carcinoma patients. Thus, decreased HSP9OAB1 expression in CRC with a poorer prognosis may represent an inadvertent bystander effect resulting from a CRC-specific selective pressure toward mTOR pathway activation as a consequence of USP22 downregulation ${ }^{6}$. In agreement with the dichotomous tumor suppressive and promoting functions of USP22, a recent review questioned the reliability of several studies describing USP22 as an exclusively oncogenic factor due to the utilization of cross-reactive antibodies used for immunohistochemical stainings as well as the lack of appropriate controls ${ }^{26}$. These data indicate that, depending on the cancer type and the biological context, USP22 inhibition could either enhance or reduce tumor growth in patients. Thus, USP22 inhibitors which have been proposed to represent promising therapeutics might not display a suitable and safe treatment option in vivo.

Since HSP90 family members are involved in the cellular adaptation to stress ${ }^{28}$, we hypothesized that diminishing HSP90 completely would result in decreased survival of USP22-deficient cells. Indeed, we demonstrated that USP22 deficiency results in increased therapeutic vulnerability towards Ganetespib in vitro and in vivo. Notably, independent of the effects of USP22 loss on proliferation (decreased in HCC1954 and increased in HCT116 siUSP22 cells), a sensitization toward HSP90 inhibition was induced. Since various HSP90 inhibitors have been successfully tested in clinical trials ${ }^{29}$, targeting HSP90 may provide an ideal approach to exploit USP22deficiency in cancer cells. Furthermore, combined 
inhibition of USP22 and HSP90 may provide an effective combinatorial approach to tumor therapy, which would overcome the context-dependency demonstrated in our studies. Interestingly, it was described that USP22 knockdown sensitized lung adenocarcinoma tumor spheres towards cisplatin treatment ${ }^{30}$. Similarly, Ganetespib-based HSP90 inhibition induced cell death via severe global chromosome fragmentation upon carboplatin treatment in vitro ${ }^{31}$. Future studies might reveal whether USP22 loss, and the subsequent reduction of HSP90 levels, will sensitize colorectal and breast cancer cells towards platinum-based therapy.

Together, in this study we demonstrate that the constitutively expressed heat shock factor HSP90AB1 is highly dependent upon USP22-mediated epigenetic regulation in human breast and colorectal cancer cell lines as well as in murine mammary and colorectal tumors. Intriguingly, in a USP22-deficient context, the further inhibition of HSP90 activity using Ganetespib effectively reduced tumor growth in vitro and in vivo. Further investigations will reveal whether USP22 expression levels can serve as a prognostic marker in cancer patients where low USP22 levels can be exploited by inducing therapeutic vulnerability to HSP90i treatment.

\section{Acknowledgements \\ We are grateful to N. Molitor and S. Beuermann and the staff of the animal facility at the European Neuroscience Institute Göttingen. Furthermore, we thank G. Salinas and F. Ludewig for performing next-generation sequencing at the Transcriptome and Genome Analysis Laboratory (TAL) Göttingen. This work was supported by funding from the Roggenbuck Foundation (to R.L.K.), the Deutsche Krebshilfe (to S.A.J.; 111600) and institutional funding provided to the Department of General, Visceral and Pediatric Surgery by the University Medical Center Göttingen (to S.A.J.). R.L.K. is supported by the Dorothea Schlözer program (University of Göttingen) and M.Z. by the Else Kroener- Fresenius Foundation.}

\section{Conflict of interest}

The authors declare that they have no conflict of interest.

\section{Publisher's note}

Springer Nature remains neutral with regard to jurisdictional claims in published maps and institutional affiliations.

Supplementary Information accompanies this paper at (https://doi.org/ 10.1038/s41419-019-2141-9).

Received: 16 August 2019 Revised: 30 October 2019 Accepted: 11 November 2019

Published online: 04 December 2019

\footnotetext{
References

1. Townsend, N. et al. Cardiovascular disease in Europe: epidemiological update 2016. Eur. heart J. 37, 3232-3245 (2016).

2. Glinsky, G. V., Berezovska, O. \& Glinskii, A. B. Microarray analysis identifies a death-from-cancer signature predicting therapy failure in patients with multiple types of cancer. J. Clin. Investig. 115, 1503-1521 (2005).

3. Glinsky, G. V. Death-from-cancer signatures and stem cell contribution to metastatic cancer. Cell cycle (Georget., Tex.) 4, 1171-1175 (2005).
}

4. Melo-Cardenas, J., Zhang, Y., Zhang, D. D. \& Fang, D. Ubiquitin-specific peptidase 22 functions and its involvement in disease. Oncotarget 7, 44848-44856 (2016).

5. Xue-Franzén, Y., Henriksson, J., Bürglin, T. R. \& Wright, A. P. H. Distinct roles of the Gcn5 histone acetyltransferase revealed during transient stress-induced reprogramming of the genome. BMC genomics 14, 479 (2013).

6. Kosinsky, R. L. et al. USP22 exerts tumor-suppressive functions in colorectal cancer by decreasing mTOR activity. Cell Death Differ. https://doi.org/10.1038/ s41418-019-0420-8 (2019).

7. Zhang, X.-Y. et al. The putative cancer stem cell marker USP22 is a subunit of the human SAGA complex required for activated transcription and cell-cycle progression. Mol. Cell 29, 102-111 (2008).

8. Melling, N. et al. Loss of H2Bub1 expression is linked to poor prognosis in nodal negative colorectal cancers. Pathol. Oncol. Res.: POR 22, 95-102 (2016).

9. Prenzel, T. et al. Estrogen-dependent gene transcription in human breast cancer cells relies upon proteasome-dependent monoubiquitination of histone H2B. Cancer Res. 71, 5739-5753 (2011).

10. Lin, Z. et al. USP22 antagonizes p53 transcriptional activation by deubiquitinating Sirt1 to suppress cell apoptosis and is required for mouse embryonic development. Mol. Cell 46, 484-494 (2012).

11. Li, Y. et al. USP22 drives colorectal cancer invasion and metastasis via epithelial-mesenchymal transition by activating AP4. Oncotarget 8, 32683-32695 (2017).

12. Liu, Y. L., Yang, Y. M., Xu, H. \& Dong, X. S. Increased expression of ubiquitinspecific protease 22 can promote cancer progression and predict therapy failure in human colorectal cancer. J. Gastroenterol. Hepatol. 25, 1800-1805 (2010).

13. Liu, Y., Yang, Y., Xu, H. \& Dong, X. Implication of USP22 in the regulation of BMI-1, c-Myc, p16INK4a, p14ARF, and cyclin D2 expression in primary colorectal carcinomas. Diagnostic Mol. Pathol.: Am. J. surgical Pathol,, part B 19, 194-200 (2010).

14. Liu, Y.-L., Yang, Y.-M., XU, H. \& Dong, X.-S. Aberrant expression of USP22 is associated with liver metastasis and poor prognosis of colorectal cancer. J. surgical Oncol. 103, 283-289 (2011).

15. Kim, D. et al. Deubiquitinating enzyme USP22 positively regulates c-Myc stability and tumorigenic activity in mammalian and breast cancer cells. J. Cell. Physiol. 232, 3664-3676 (2017).

16. Zhang, Y. et al. Elevated expression of USP22 in correlation with poor prognosis in patients with invasive breast cancer. J. cancer Res. Clin. Oncol. 137, 1245-1253 (2011)

17. Schneider, D. et al. The E3 ubiquitin ligase RNF40 suppresses apoptosis in colorectal cancer cells. Clin. epigenetics 11, 98 (2019).

18. Subramanian, A. et al. Gene set enrichment analysis: a knowledge-based approach for interpreting genome-wide expression profiles. Proc. Natl Acad. Sci. USA 102, 15545-15550 (2005).

19. Kosinsky, R. L. et al. Loss of RNF40 decreases NF-KB Activity in colorectal cancer cells and reduces colitis burden in mice. J. Crohn's colitis 13, 362-373 (2019).

20. Kosinsky, R. L. et al. Usp22 deficiency impairs intestinal epithelial lineage specification in vivo. Oncotarget 6, 37906-37918 (2015).

21. Dymecki, S. M. Flp recombinase promotes site-specific DNA recombination in embryonic stem cells and transgenic mice. Proc. Natl Acad. Sci. USA 93, 6191-6196 (1996).

22. Uhlen, M. et al. A pathology atlas of the human cancer transcriptome. Science. https://doi.org/10.1126/science.aan2507 (2017).

23. Haase, M. \& Fitze, G. HSP90AB1: helping the good and the bad. Gene $\mathbf{5 7 5}$ 171-186 (2016)

24. Dutta Gupta, S., Bommaka, M. K. \& Banerjee, A. Inhibiting protein-protein interactions of Hsp90 as a novel approach for targeting cancer. Eur. J. medicinal Chem. 178, 48-63 (2019).

25. Muller, W. J., Sinn, E., Pattengale, P. K., Wallace, R. \& Leder, P. Single-step induction of mammary adenocarcinoma in transgenic mice bearing the activated c-neu oncogene. Cell 54, 105-115 (1988).

26. Jeusset, L. M.P. \& McManus, K. J. Ubiquitin specific peptidase 22 regulates histone $\mathrm{H} 2 \mathrm{~B}$ mono-ubiquitination and exhibits both oncogenic and tumor suppressor roles in cancer. Cancers. https://doi.org/10.3390/cancers9120167 (2017).

27. McDowell, C. L., Bryan Sutton, R. \& Obermann, W. M. J. Expression of Hsp90 chaperone corrected proteins in human tumor tissue. Int. J. Biol. macromolecules 45, 310-314 (2009). 
28. Whitesell, L. \& Lindquist, S. L. HSP90 and the chaperoning of cancer. Nat. Rev. Cancer 5, 761-772 (2005).

29. Solárová, Z., Mojužšš, J. \& Solár, P. Hsp90 inhibitor as a sensitizer of cancer cells to different therapies (review). Int. J. Oncol. 46, 907-926 (2015).

30. Yun, X. et al. Targeting USP22 suppresses tumorigenicity and enhances cisplatin sensitivity through ALDH1A3 downregulation in cancer-initiating cells from lung adenocarcinoma. Mol. cancer Res.: MCR 16, 1161-1171 (2018).

31. Kramer, D. et al. Strong antitumor synergy between DNA crosslinking and HSP90 inhibition causes massive premitotic DNA fragmentation in ovarian cancer cells. Cell death Differ. 24, 300-316 (2017). 